\title{
Johann Adam Schall von Bell - Ein Kölner des 17. Jahrhunderts in China
}

\section{Zhu Jianbua}

\section{Stationen im Leben des Johann Adam Schall von Bell}

Vor dem Hintergrund frühneuzeitlicher Globalisierung ist die deutsch-chinesische Fachkommunikation bzw. Fachsprachenforschung zweifelsohne auf den wissenschaftlichen Austausch und den Wissenstransfer zwischen Ost und West im 17. Jahrhundert zurückzuführen, als die Jesuiten zur Ausübung ihrer Missionsarbeit vielfach die Vermittlung von Fachwissen als Instrument nutzten. So fungierte etwa Adam Schall von Bell als Vertreter der westlichen Fachwelt am Kaiserhof, wiewohl er in erster Linie kein Wissenschaftler war. Durch folgende biografische Darstellungen wird der Verfasser versuchen zu demonstrieren, warum und wie Adam Schall von Bell in der chinesischen Kaisergeschichte als Kulturheld verehrt und heroisiert wurde und welche Ansatzpunkte diese historische Figur für heutiges Forschungsinteresse bietet.

Johann Adam Schall von Bell wurde am 1. Mai des Jahres 1592 in Köln (oder Lüftelberg) geboren. ${ }^{1} 1603$ trat er in das von Jesuiten geleitete Collegium Tricoronatum, das Dreikronen- oder Dreikönigsgymnasium in Köln ein, wo er klassische Bildung in den Fächern Latein, Religion und Geschichte, Deutsch, Logik, Rhetorik und Metaphysik genoss. Ab 1608 studierte er in Rom an dem von Jesuiten geleiteten Collegium Germanicum, das 1552 durch Papst Julius III. gegründet worden war. ${ }^{2}$ Drei Jahre später, am 21 . Oktober 1611, trat er in den Jesuitenorden ein. Er hatte neben Theologie auch Mathematik und Astronomie studiert. 1613 wurde Adam Schall von Bell zu theologischen Studien an der Gregoriana aufgenommen. Dort traf er mit Europäern zahlreicher Nationalitäten zusammen. Möglicherweise reizte ihn die internationale Atmosphäre des Noviziatshauses der römischen Ordensprovinz zu St. Andrea.

Am 2. Januar 1616 bat er den General des Jesuitenordens, Mutius Vitelleschi, ihn ins östliche Indien, möglichst sogar nach China zu schicken. Dieser Wunsch ging zwei Jahre später, 1618, in Erfüllung. Nach einer abenteuerlichen Seefahrt von Lissabon aus erreichte Adam Schall das portugiesische Macao. Von diesem europäischen Stützpunkt aus, dem einzigen im chinesischen Kaiserreich, wo er intensiv Chinesisch lernte und Vorbereitungen für seine Chinamission traf, sollte

1 Li Lanqin (李兰琴): 汤若望传 (Biographie des Tang Ruo Wang), Dong Fang Chubanshe 1995, S. 3.

2 Da Subin (达素涁): “通玄教师”汤若望 (Meister himmlischer Geheimnisse), übers. von Ernst Stürmer (1980), Peking 1989, S. 9-10. 
Adam Schall mit sieben weiteren angereisten Jesuiten im Auftrag des Vatikan im großen China die Christianisierung vorantreiben. ${ }^{3}$

$1623 \mathrm{kam}$ er in Peking an. Dort vertiefte er seine Chinesischkenntnisse und nahm Kontakte zu chinesischen Gelehrten und Beamten auf. Der bedeutendste darunter war Dr. Paul Xu Guangqi (Hsü Kuang-ki), ein berühmter Wissenschaftler und Gelehrter in der Ming-Dynastie und Kultusminister zu jener Zeit (礼部 尚书兼文渊阁大学士). ${ }^{4}$ Bekannt wurde Adam Schall von Bell durch seine astronomischen Kenntnisse. So sagte er etwa die Mondfinsternis am 8. Oktober 1623 voraus und wurde in der Folge von vielen hohen chinesischen Beamten verehrt. 1630 wurden Adam Schall und sein Begleiter Giacomo Rho ins Kalenderamt berufen. Auf Empfehlung Xu Guangqis wurde Schall von Bell von Kaiser Chungcheng empfangen. Weil der Kalender große Bedeutung als Legitimitätsbeweis für die Herrschaft des Kaisers und seiner Dynastie hatte, erhielt Adam Schall den Auftrag, den chinesischen Kalender den neuesten Kenntnissen aus Europa anzupassen und ihn nach westlichem Vorbild zu reformieren. Dafür musste er seinen Namen ändern. Dem Rat seines Freundes Pater Longobardi folgend, nannte er sich von nun an Tang-Jo-wang. ${ }^{5}$

In den folgenden sieben Jahren veröffentlichten Pater Longobardi, Johannes Terrentius, Giacomo Rho und Adam Schall, zusammen mit chinesischen Astronomen, auf Chinesisch Bücher über Mathematik und Naturwissenschaften und berechneten einen genauen Kalender, der die Position der Sterne für jeden Tag anzeigte. Das Gesamtwerk umfasste rund 150 Bändchen in drei Abteilungen: Hilfswissenschaften, astronomische Theorie und Praxis, astronomische Tafeln. Die meisten Bücher wurden vom Minister Dr. Paul Xu Guangqi unter Mitwirkung von Li Shih-tsao und Li Tien-king sprachlich überarbeitet und zu einer Sammlung, einer Enzyklopädie der europäischen Wissenschaft vereinigt. Zu Ehren des Kaisers erhielt die Sammlung den Titel Astronomische Bücher Chung Chengs (崇祯历书). Sammlung von Abhandlungen über Astronomie und Kalenderkunde der Chungcheng-Regierungsperiode 1629-1634. Später erschien sie unter folgenden anderen Titeln: Sin-fa-suan-schu 新法算书 und Si-yang-fa-sin-shu 西洋法新书 sowie Shi-xian-li 时宪历.

Um die Jesuiten, insbesondere Adam Schall, zu belohnen, schenkte der Kaiser den Missionaren eine Ehrentafel, deren Inschrift lautet: „Ich, der Kaiser, lobe und schütze die Lehre vom Himmel 钦裹天学 $1639^{\text {“. } .6}$

Am 23. April 1644 marschierte die Armee von Li Zicheng (李自成) in Peking ein, die Ming-Dynastie wurde gestürzt. Nach einem Monat wurde diese Armee von den Mandschuren wieder aus der Stadt getrieben, welche daraufhin in der

3 Alfons Väth: Johann Adam Schall von Bell, SJ. Missionar in China, kaiserlicher Astronom und Ratgeber am Hofe von Peking 1592-1666, Nettetal 1991, S. 5-6. Außerdem: Li Lanqin (李兰琴): 汤若望传 (Anm. 1), S. 13-14.

4

Ebd.

Ebd., S. 3.

6 Ebd., S. 42. 
Stadt ihr Quartier nahmen. Am 30. Oktober wurde Peking zur Hauptstadt einer neuen Dynastie - der Qing-Dynastie (1644-1912) - erklärt. Adam Schall von Bell erlebte den Untergang der Ming-Dynastie in der Hauptstadt und blieb während des Machtwechsels in der Residenz der Missionare. Er konnte den jungen Kaiser Shunzhi und dessen Regenten davon überzeugen, seine Kenntnisse zu ihrem Nutzen zu verwenden, da auch die neue Dynastie zur Legitimation ihrer Herrschaft die europäischen Astronomen zur Berechnung eines korrekten Kalenders benötigte. So wurde Adam Schall 1644 zum Direktor des Astronomischen Amtes (钦天 监正) in Peking ernannt. Durch seine hervorragende Arbeit im Astronomischen Amt gelangte er zu hohen Ehren. Im Laufe der nächsten Jahre wurde er persönlicher Berater des jungen Kaisers. Er erreichte den höchstmöglichen Beamtenrang. 1653 wurde ihm von Kaiser Shunzhi die Auszeichnung ,Die Geheimnisse des Himmels ergründender Lehrer' (Tongxuan-jiaoshi 通玄教师) verliehen. 1658 wurde er zum ,Hohen Würdenträger der Kaiserlichen Bankette' (Kuang-lu tai-fu 光禄 大夫) ernannt, womit sich der Rang eines Staatsbeamten der 1. Klasse (Mandarin) verband. ${ }^{7}$

Nachdem 1661 Kaiser Shunzhi gestorben war, geriet Adam Schall allmählich in Schwierigkeiten. Einerseits war der Ritenstreit infolge einer dominikanischen Visitation in Peking wieder entfacht worden. Der Jesuit wurde in Rom angeklagt. Auch seine politisch-wissenschaftlichen Ämter lösten erhebliche Kritik aus: Im Vatikan verstärkte sich der Standpunkt, Jesuiten sollten eigentlich keine weltlichen Ämter annehmen. ${ }^{8}$

Andererseits fiel Adam Schall im Jahre 1664 durch Intrigen einiger Hofbeamter auch am Kaiserhof in Ungnade. Aufgrund der Anklage des Hofbeamten Yang Guangxian war er ins Gefängnis geworfen worden und wurde wegen Hochverrats und der Verbreitung falscher Religion sowie falscher astronomischer Lehren zum Tode durch Lingchi (凌迟), Zerstückelung bei vollem Bewusstsein, verurteilt. Aber das Todesurteil vom 15. April 1665 wurde nicht vollstreckt, denn durch eine Reihe schrecklicher Naturkatastrophen, wie Erdbeben und einem Brand im Palast, glaubten die Leute, dass der Himmel mit dem Urteil über Adam Schall nicht einverstanden sei und also er unschuldig sei. Adam Schall von Bell durfte am 15. Mai 1665 in die Jesuitenresidenz Südhalle (Nantang 南堂) zurückkehren. Aber die Gefangenschaft hatte seine Gesundheit schwer geschädigt. Am 15. August 1666 starb der neben Matteo Ricci und Ferdinand Verbiest bedeutendste Mann der Jesuitenmission, nachdem er 47 Jahre in China verbracht hatte. 1668 ließ der zweite Kaiser der Qing-Dynastie, Kangxi, Johann Adam Schall von Bell rehabilitieren und ihm ein Ehrenbegräbnis auf dem Jesuitenfriedhof in Shala zuteilwerden. ${ }^{9}$ Die Astronomiearbeit der Europäer konnte sich erneut durchsetzen und die Missionsarbeit nach und nach wieder aufgenommen werden.

7 Da Subin (达素涁): “通玄教师”汤若望 (Anm. 2), S. 70-72.

8 Anton Huonder: Der chinesische Ritenstreit, Aachen 1921, S. 28.

9 Li Lanqin (李兰琴): 汤若望传 (Anm. 1), S. 169. 


\section{Johann Adam Schall von Bell als politische Figur}

Adam Schall von Bell war in erster Linie ein Missionar und Gesandter der katholischen Kirche und des Jesuitenordens aus Rom. Für seine christliche Mission stellen die folgenden Werke seine bedeutendsten im Feld der Religion dar:

1) Von der göttlichen Vorsehung (Chu-chib-kün-cheng 主制群徵 ), 1629

2) Die Acht Seligkeiten (Chen-fu-hsün-chuan 真福训诠), 1634

3) Über das Bild des Erlösers (Tsin-cheng-shu-siang 进呈书像), 1640

4) Beispiele von Heiligen der katholischen Kirche (Chung-yi-tang-jib-ki-sui-pi 崇一堂日 记随笔)

5) Über den Ursprung des Christentums (Chu-kiao-yüan-ki 主教缘起) $1643^{10}$

Den quantitativ höheren Anteil seiner Arbeit machen jedoch Adam Schalls wissenschaftliche Werke aus.

Während seiner Karriere wurde er politisch immer wieder scharf aus den eigenen Reihen kritisiert. So wurde Adam Schall in Rom wegen Unterstützung des Aberglaubens durch die Ausarbeitung des Volkskalenders angeklagt. ${ }^{11}$ Seine Stellung als Direktor des Astronomischen Amtes erregte Kritik im Orden, da es Jesuiten nicht erlaubt war, weltliche Ämter und Ehren anzunehmen. Auf der anderen Seite sah sich Adam Schall auch zahlreichen Anfeindungen einflussreicher Chinesen ausgesetzt, die der Verbreitung des Christentums in China feindlich gegenüberstanden. Dadurch wurde Schall von Bell zunehmend zu einer umstrittenen Person am chinesischen Kaiserhof. Und dies führte schließlich zur Verhaftung des Jesuiten und seiner Mitstreiter.

\section{Beziebungen zu chinesischen Beamten und Gelehrten}

Schon als Adam Schall zum ersten Mal in Peking ankam, begann er freundschaftliche Beziehungen zu vielen chinesischen Gelehrten zu knüpfen. Dahinter steckte zum Teil das Kalkül, dass die Freundschaft mit hohen und höchsten Beamten die Sicherheit der Mission garantieren könne. Diese Akkommodation war daher Teil der Strategie hinter der Missionsarbeit der Jesuiten in China. Durch seine stets warmherzige Fürsorge und Hilfe für chinesische Christen konnte Adam Schall in diesen Kreisen gute Beziehungen aufbauen; durch seine fachkundige Arbeit wiederum gelang es ihm, das Vertrauen chinesischer Gelehrter zu gewinnen.

Dr. Paul Xu Guangqi war einer der wichtigsten chinesischen christlichen Mandarine der Ming-Dynastie. Die Beziehung zwischen ihm und Adam Schall war in gewisser Hinsicht wie zwischen Meister und Schüler. Adam Schall lernte nach der Ankunft in Peking zunächst bei ihm Chinesisch und trat später auf Xu Guangqis

10 Ebd., S. 134-136.

11 Vgl. Huonder: Ritenstreit (Anm. 8), S. 28. 
Empfehlung ins Astronomische Amt ein. Als Direktor des Astronomischen Amtes kümmerte sich Xu Guangqi geradezu fürsorglich um Adam Schall und andere Jesuiten; entsprechend eng gestaltete sich die Zusammenarbeit. Kurz vor Xu Guangqis Tod war Adam Schall stets an seiner Seite und spendete ihm religiöse Tröstung. ${ }^{12}$

Im Umgang mit Chinesen soll er durchweg freundlich, warmherzig, hilfsbereit und offen gewesen sein. ${ }^{13}$ Sein eheloses Leben war ein Rätsel für viele Chinesen. Nach chinesischer Sitte galt es als große Schande für die Vorfahren, keine Nachkommen zu haben. Auf Nachfrage erklärte Adam Schall, in seiner Heimat sei es gleichermaßen Sitte, dass Menschen heiraten, Familien gründen und Kinder haben, doch ein gottgeweihter Mensch müsse auf seine persönlichen Begierden und Familienpflichten verzichten, um sich ganz Gott hingeben zu können.

\section{Johann Adam Schall von Bell als ein Fachsprachen-, Fachwissen- und Fachkulturenvermittler}

Adam Schalls wissenschaftliche Tätigkeit war überaus produktiv und brachte zahlreiche Fachdokumente, Publikationen und Übersetzungen in Zusammenarbeit mit chinesischen Fachleuten hervor. Ein Großteil seiner Werke behandelt mathematisch-astronomische Theorie. Neben Adam Schalls neuem Kalender, welcher durch Dr. Paul Xu Guangqi dem Kaiser überantwortet wurde, sind folgende Schriften von Adam Schall übersetzt oder zusammengefasst worden:

1) Neue Methode der europäischen Astronomie (Si-yang-sin-fa-li-shu 西洋新法历书)

2) Lebre von den Sonnen- und Mondfinsternissen (Kiao-shib-li-chib 交食历指)

3) Tafeln der Sonne- und Mondfinsternis (Kiao-sbib-piao 交食表)

4) Erörterung über die Finsternisse vom Altertum bis zur Gegenwart (Ku-kin-kiao-shibkao 古今交食考)

5) Tafeln über den Auf- und Niedergang der Fixsterne (Heng-sing-chu-mu-piao 恒星 出没表)

6) Fixsterntafeln (Heng-sing-piao 恒星表)

7) Einfübrung in die Astronomie (Sin-fa-li-yin 新法历引)

8) Unterschiede der neuen Astronomie zur alten (Sin-fa-piao-yi 新法表异)

9) Die Berechnung der Finsternisse (Cheh-shib-leo 测食略)

10) Über die Fixsterne (Heng-sing-li-chib 恒星历指)

11) Abhandlung über den Himmels- und Erdglobus (Hun-tian-yi-shuo 浑天仪说)

12) Trigonometrische Tafeln (Pa-sien-piao 八线表)

13) Geschichte der Astronomie des Abendlandes (Li-fa-si-chuan 历法西传)

14) Lösung der Zweifel bezüglich der neuen Methode [der Kalenderbearbeitung] (Sinli-bsiao-buo 新历晓惑)

12 Li Lanqin (李兰琴): 汤若望传 (Anm. 1), S. 73.

13 Ebd., S. 77-78. 
15) Antworten aufZweifel bezüglich der Kalender (Min-li-pu-chu-kiai-huo 民历补注解 惑)

16) Bearbeitung des Kalenders nach der europäischen Methode (Si-zang-cheh-jib-li 西洋 测日历)

17) Kurze Erklärung zum Verständnis des Kalenders (Hsio-li-siao-pien 学历小辩)

18) Bittgesuche, Denkschriften und Antworten (Tsou-shu 奏疏)

19) Der Volkskalender und der Planetenkalender (Shin-fa-min-li u. Tsi-cheng-Shi-cheng-li 西 法民历）

20) Anweisung über den Gebrauch der astronomischen Instrumente (Chu-ki-yung-fa 诸器 用法)

21) Große Himmelskarte (Sing-tu 星图), acht große Blätter von je $175 \times 65 \mathrm{~cm}$

22) Karte der sichtbaren Sterne (Sien-kiai-tsung-sing-tu 见界总星图)

23) Äquatorialsternkarte (Huang-tao-tsung-sing-tu 黄道总星图)

24) Methode des Rechnens mit Rechenstäbchen (Chou-suan 筹算)

25) Trigonometrie (Ta-cheh 大测)

26) Abriss der Astronomie (Chegh-tien-zo-shuo 测天约说) $)^{14}$

$\mathrm{Zu}$ seinen großen Leistungen gehört außerdem die Übertragung von Georgius Agricolas (1494-1555) De re metallica (1556) ins Chinesische, welche von Adam Schall auf Initiative des chinesischen Beamten, Gelehrten und Astronomen Li Tianjing 李天经 (1579-1659) mit Unterstützung der Praktikanten Yang Zhi-hua 杨之华 und Huang Hongxian 黄宏宪 in den Jahren 1638-1640 erstellt wurde. Die lange verschollen geglaubte Schrift Kunyu-gezhi (坤宇格致) wurde erst 2015 in der Stadtbibliothek von Nanjing wiederentdeckt. ${ }^{15}$

\section{Johann Adam Schall von Bell als Freund und Berater des chinesischen Kaisers}

Im Jahre 1644 kam der siebenjährige Shunzhi an die Macht. Er war der erste Kaiser der Qing-Dynastie. Adam Schall wurde im Laufe der nächsten Jahre persönlicher Berater des jungen Kaisers. Er gelangte zu hohen Ehren und wurde von Kaiser Shunzhi zum Lehrer und Mentor ernannt; in dieser Funktion genoss Adam Schall sein Vertrauen. Zahlreiche Gunsterweisungen bezeugten den ihm entgegengebrachten Respekt: „Er pflegte Schall ,Ma-Fa' zu nennen. Das Mandschuwort bedeutet ,Ehrwürdiger Vater ${ }^{\varsigma}$ und schließt in sich alle Liebe und alles $\mathrm{Zu}$ trauen eines Sohnes zu seinem Vater, eines Schülers gegenüber seinem Meister ein. Der Vater und der Meister war zuweilen streng; aber der junge Herrscher hat

14 Ebd., S. 32-34.

15 Vgl. Hans Ulrich Vogel: Sensationelle Wiederentdeckung eines Manuskriptes: Die chinesische Übersetzung von Georgius Agricolas De re metallica aus dem Jahre 1640, S. 1, www. academia.edu/16465520/, 20. Januar 2020. 
es nicht übel genommen und blieb ein gelehriger Schüler." ${ }^{\text {16 }}$ Nach Väth verhielt sich das persönliche Verhältnis zwischen Shunzhi und Ma-Fa wie eine VaterSohn-Beziehung. Nach Meinung des Verfassers glich es eher der Beziehung zwischen Großvater und Enkel, war doch zum Zeitpunkt seiner Machtübernahme der Kaiser noch keine dreizehn Jahre alt, Adam Schall hingegen schon in seinem 59. Lebensjahr. Zuvor hatte schon die Kaiserinmutter Xiaozhuang Adam Schall mit der Ehrenbezeichnung, Adoptivvater versehen. Bei Adam Schall von Bell lernte der Kaiser nicht nur Sternkunde, sondern empfing auch Unterweisung auf dem Weg zu einem guten, tugendhaften Menschen und erfolgreichen Kaiser. Adam Schall hatte den Mut und die Autorität, dem Kaiser den Weg zu weisen. ${ }^{17}$

In den Jahren 1656 und 1657 kam der Kaiser 24-mal zu längerem Aufenthalt nach Nantang (南堂). Er benahm sich bei Adam Schall wie ein Freund. Die Verleihung der Ehrentitel an Adam Schall und dessen Vorfahren fand am 2. Februar 1658 statt. $^{18}$

\section{Fazit}

Nach der Betrachtung der verschiedenen Biografien kommt der Verfasser zur Schlussfolgerung, dass Johann Adam Schall von Bell in dem Willen, das Christentum zu verbreiten, nach China gereist war und sein Leben seiner Missionsarbeit gewidmet hat. Seine größte Ambition dürfte gewesen sein, den Kaiser zum christlichen Glauben zu bekehren.

Tatsächlich war Schall von Bell jedoch vor allem in der interkulturellen Fachkommunikation und als bedeutender Vermittler zwischen westlichen und östlichen Fachkulturen sehr erfolgreich. Durch seine fachlichen Tätigkeiten, auch wenn er sie anfangs zum Zwecke seiner Missionstätigkeit einsetzte, gewann er seine epochale Bedeutung als Schlüsselfigur des west-östlichen Kulturtransfers und wurde daher vom chinesischen Kaiser und von chinesischen Gelehrten in der Ming- und QingDynastie als Kulturheld vielfach geehrt. In Deutschland wurden die Erfolge von Schall von Bell erst mit Verspätung gewürdigt. Sowohl in Lüftelberg als auch in Köln wurden Denkmäler für Schall von Bell errichtet. Zu seinem 400. Geburtstag gab die Bundespost eine Sonderbriefmarke heraus.

Bis heute gilt seiner wissenschaftlichen Arbeit großes Forschungsinteresse, da die Leistungen, die Adam Schall im Zusammenwirken mit seinen Ordensbrüdern und chinesischen Gelehrten in der Ming- und Qing-Dynastie vollbrachte, gerade im heutigen Zeitalter der Globalisierung aufschlussreich und weiterhin erforschenswert sind. ${ }^{19}$

16 Väth: Johann Adam Schall (Anm. 3), S. 172.

17 Li Lanqin (李兰琴): 汤若望传 (Anm. 1), S. 94.

18 Ebd., S. 185.

19 Zhu Jianhua / Guo Yiwei: Perspektiven der interkulturellen Fachkommunikation, in: Jianhua Zhu / Rudolf Hoberg (Hg.): Germanistische Sprachwissenschaft und Deutschunterricht 
Über die Fachsprachen und deren Anwendung in der Fachkommunikation, die auf den damaligen Fachkulturen in der Zeit der Vormodernisierung und der frühzeitlichen Globalisierung basieren, fehlen fast in allen Biografien ausreichende Darstellungen aus fachsprachenlinguistischer Sicht; etwa die Einführung von Fachbegriffen und -bezeichnungen, Fachübersetzungen in der damaligen Zeit usw. Jedoch bieten gerade die zahlreichen Werke und Dokumente von und über Johann Adam Schall von Bell wichtige Ansatzpunkte, um Fachsprachen und Fachkulturen in der deutsch-chinesischen Fachkommunikation der Frühen Neuzeit weiter zu erforschen.

in chinesisch-deutscher Perspektive. Frankfurt am Main 2010, S. 257-268, hier S. 267. AuBerdem: Zhu Jianhua / Chen Chen: 德意志语言思想背景下的专用语研究。(朱建华, 陈 忱) 西安外国语大学学报, 2015 年第2期35-38页。(Fachsprachenforschung im Hintergrund des deutschen Sprachdenkens), in: Journal of Xian International Studies University 2, 2015, S. 35-38, hier S. 36-37. 